Supporting Information

\title{
ZnO-Based Nanoplatforms for Labeling and Treatment of \\ Mouse Tumors without Detectable Toxic Side Effects
}

Dai-Xin Ye, Ying-Ying Ma, Wei Zhao, Hong-Mei Cao, Ji-Lie Kong, ${ }^{*}$ Huan-Ming

Xiong $^{*}$ and Helmuth Möhwald

E-mail: jlkong@,fudan.edu.cn or hmxiong@,fudan.edu.cn

Figures and Captions

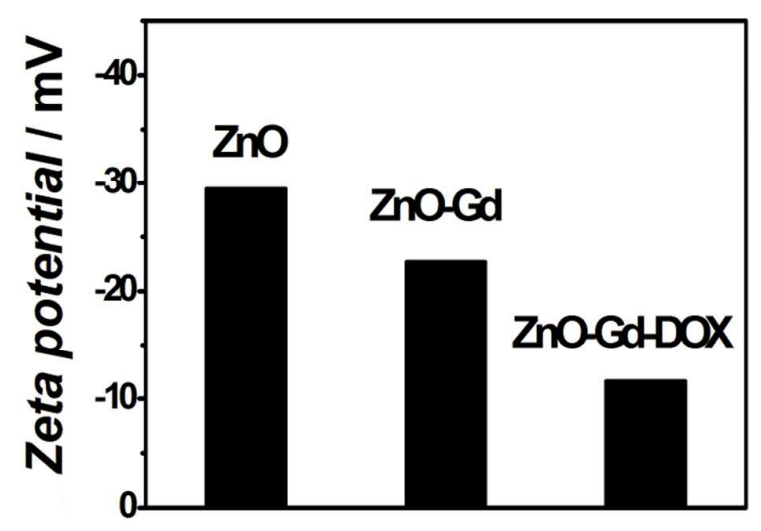

Figure S1. Zeta potentials of $\mathrm{ZnO}, \mathrm{ZnO}-\mathrm{Gd}$ and $\mathrm{ZnO}-\mathrm{Gd}-\mathrm{DOX}$ NPs in water.

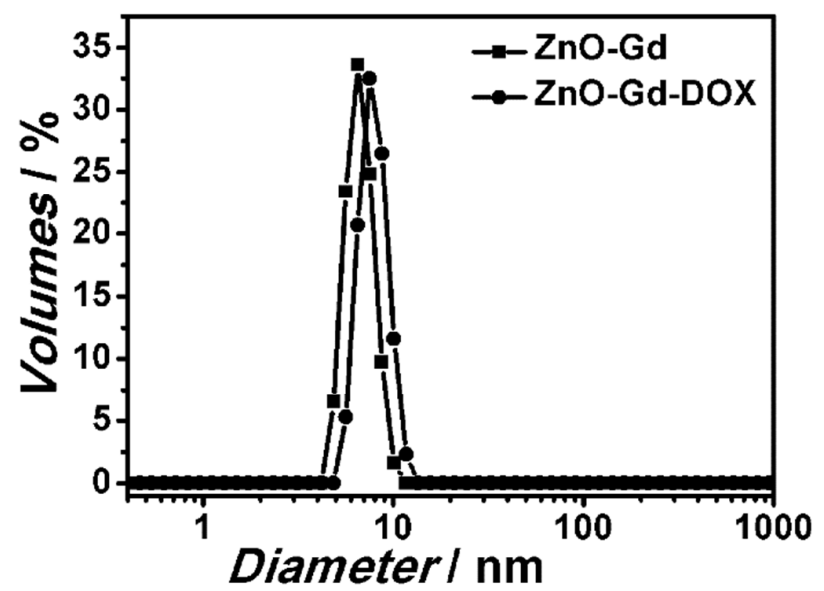

Figure S2. Dynamic light scattering (DLS) data of $\mathrm{ZnO}-\mathrm{Gd}$ and $\mathrm{ZnO}-\mathrm{Gd}-\mathrm{DOX}$ NPs in water. 


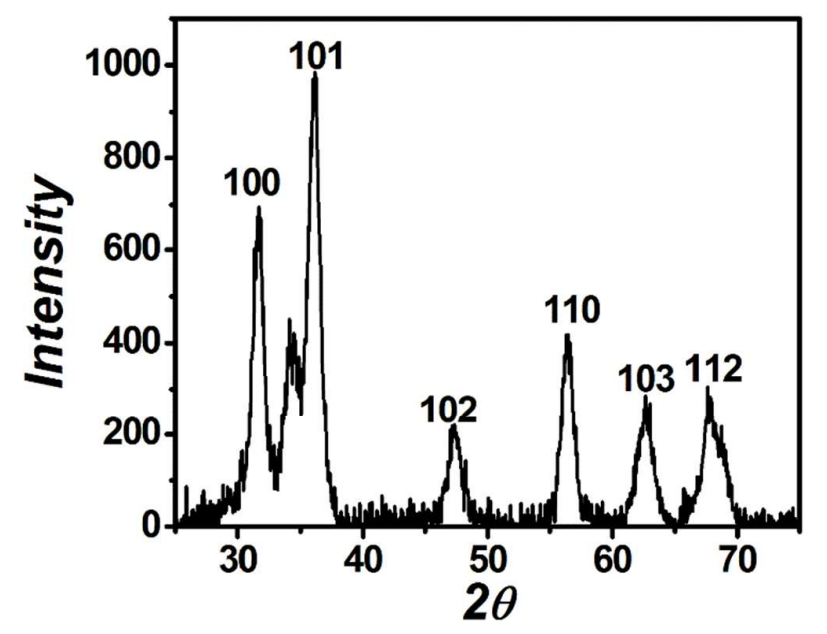

Figure S3. X-ray diffraction patterns of ZnO-Gd NPs.

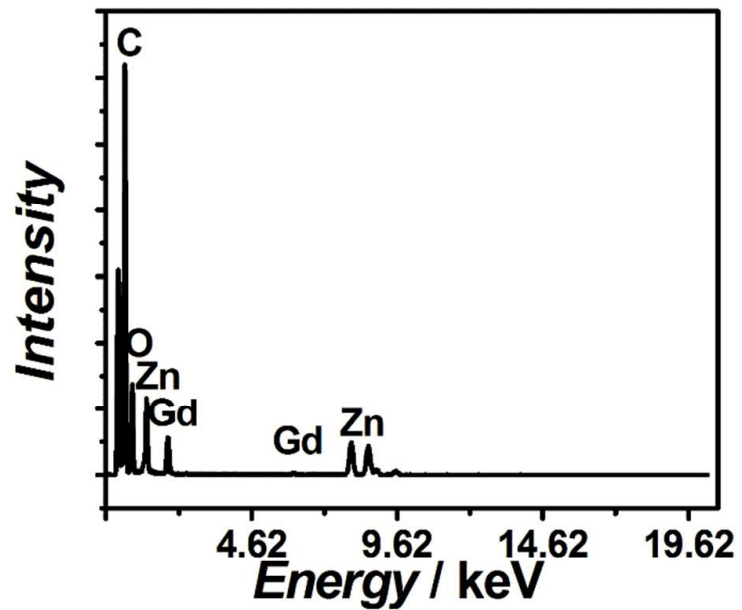

Figure S4. Energy Dispersive X-ray (EDX) spectrum of ZnO-Gd NPs.

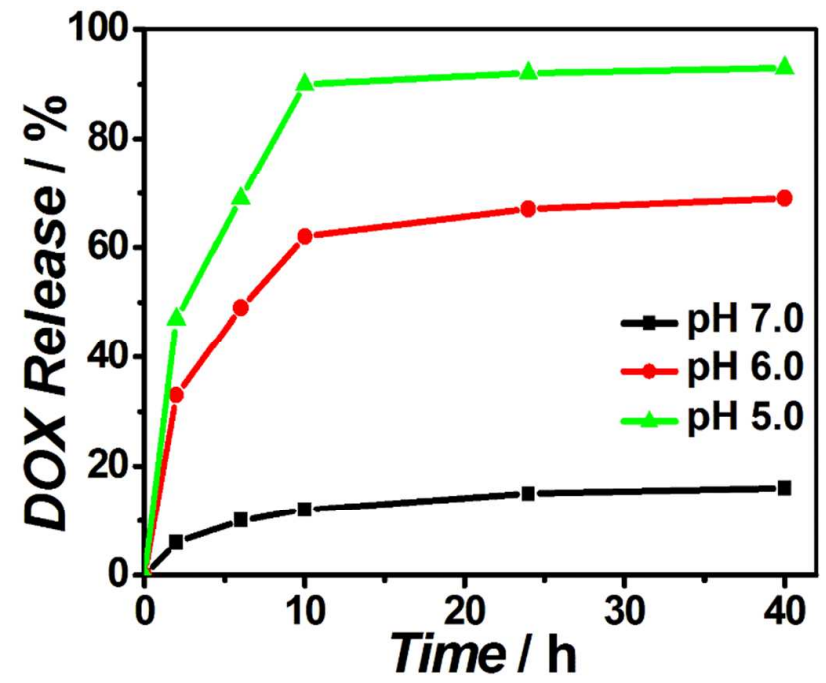

Figure S5. DOX release profile of ZnO-Gd-DOX NPs at different $\mathrm{pH}$ values. 


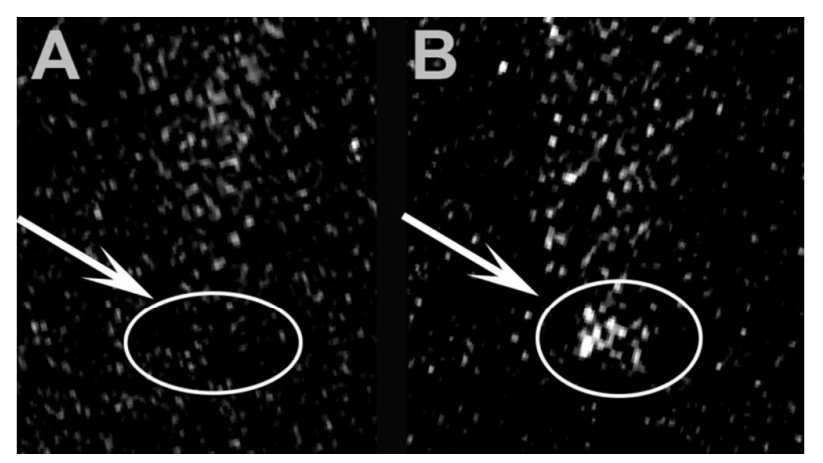

Figure S6. $\mathrm{T}_{1}$-weighted images of (A) blank BxPC-3 cells and (B) BxPC-3 cells incubated with $\mathrm{ZnO}-\mathrm{Gd}-\mathrm{DOX} \mathrm{NPs}$ (corresponding to $0.06 \mathrm{mM}$ of $\mathrm{Gd}^{3+}$ ions) for $1.5 \mathrm{~h}$. The marked area in (B) is evidently brighter than that in (A), which shows the precipitation of cells in a centrifuge tube after centrifugation, indicating that ZnO-Gd-DOX NPs can serve as effective MRI contrast agent.

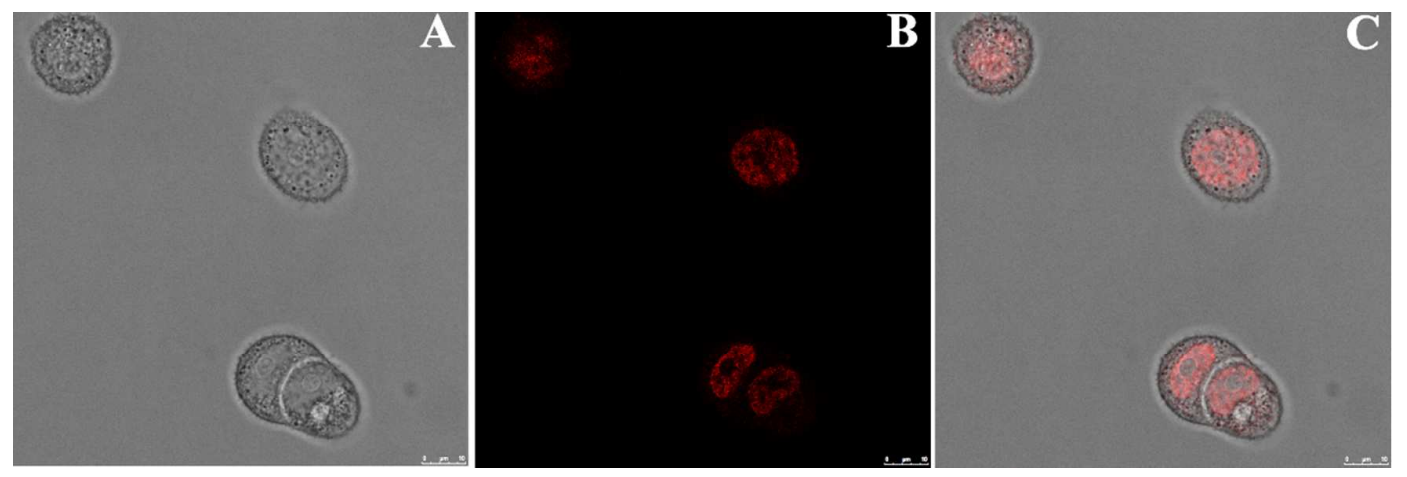

Figure S7. After incubation with ZnO-Gd-DOX NPs for $1.5 \mathrm{~h}$ at $37{ }^{\circ} \mathrm{C}$, the CLSM images of BxPC-3 cells in (A) bright field, (B) fluorescence field under $488 \mathrm{~nm}$ of excitation, and (C) the overlay of (A) and (B). Each scale bar represents $10 \mu \mathrm{m}$. It is apparent, that the $\mathrm{ZnO}-\mathrm{Gd}-\mathrm{DOX}$ NPs have entered the cells and decomposed to release DOX, and the DOX has penetrated into the nuclei. However, the nucleoli inside the nuclei are not stained by DOX. 


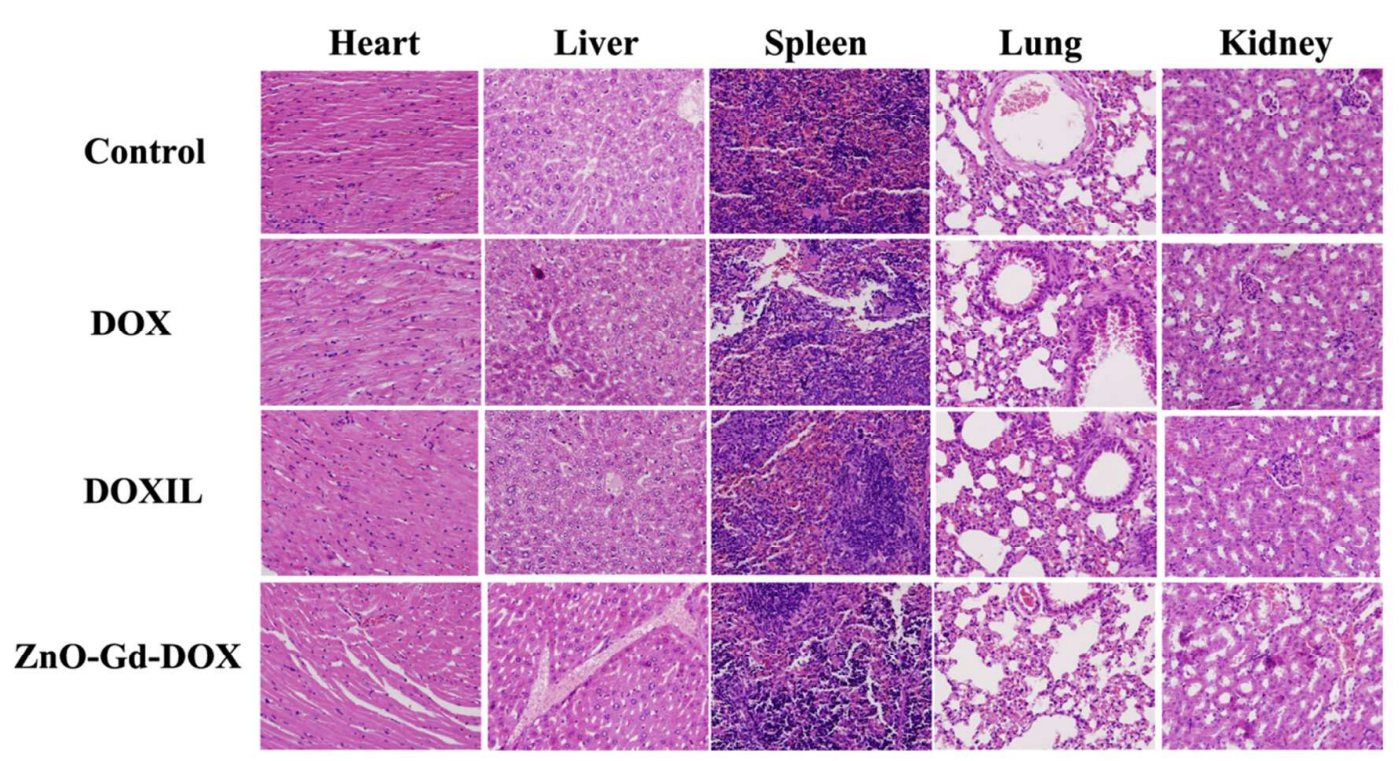

Figure S8. H\&E stained images of major organs collected from the tumor-bearing mice treated with different agents (DOX, DOXIL and ZnO-Gd-DOX are at the DOX dosage of $2 \mathrm{mg} / \mathrm{kg}$; Control is injected with $200 \mu \mathrm{L}$ of saline).
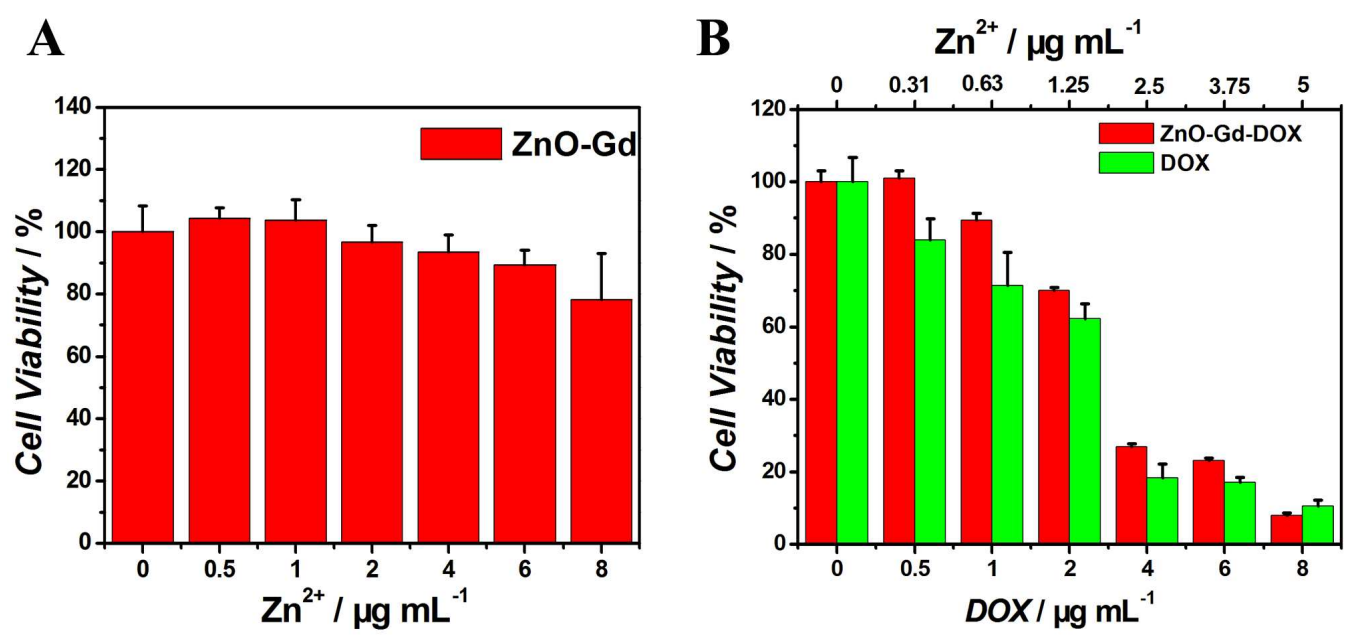

Figure S9. Viability of the BxPC-3 cells after $24 \mathrm{~h}$ incubation with (A) ZnO-Gd NPs, (B) ZnO-Gd-DOX NPs and DOX, respectively. 

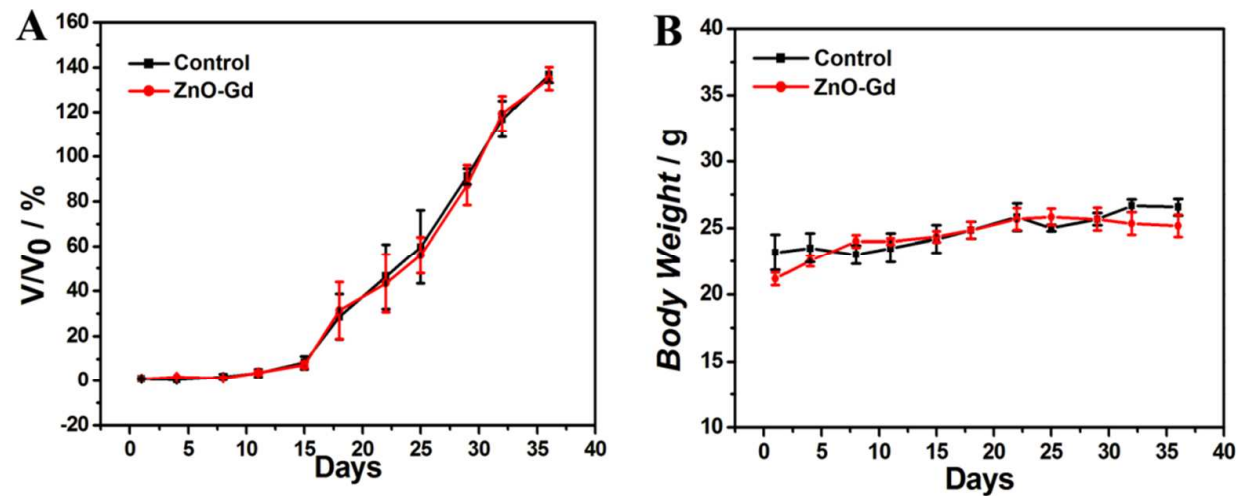

Figure S10. (A) Tumor growth curves and (B) Body weight evolutions of the BxPC-3 bearing mice with $\mathrm{ZnO}-\mathrm{Gd}$ solution and saline (control) injected, respectively. The dosage of $\mathrm{ZnO}-\mathrm{Gd}$ is $1.25 \mathrm{mg} / \mathrm{kg}$ of $\mathrm{Zn}^{2+}$ ions in each injection.

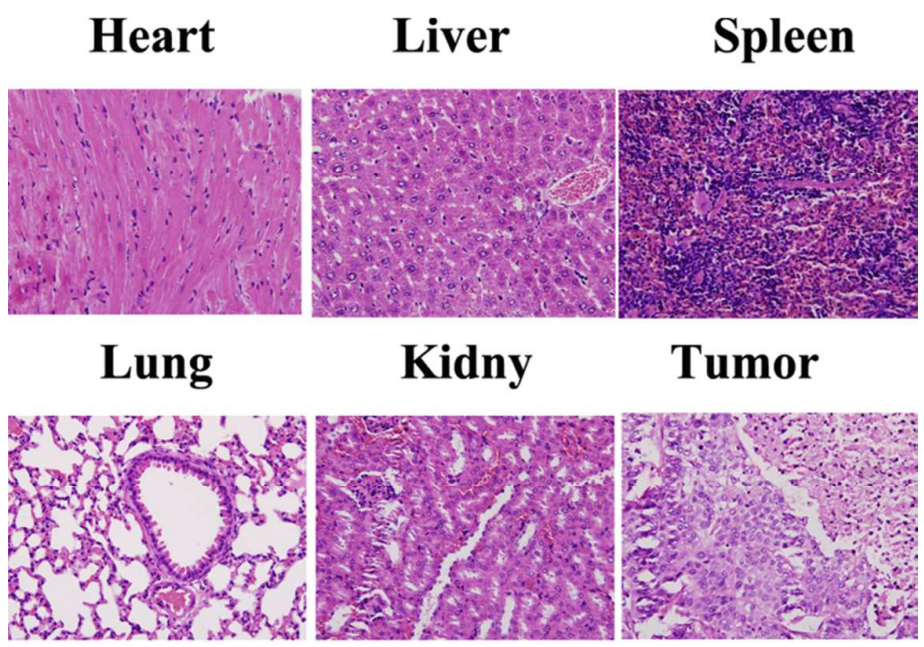

Figure S11. H\&E stained images of the major organs collected from the tumor-bearing mice after 36 days' treatment by $\mathrm{ZnO}-\mathrm{Gd}$ solution with $1.25 \mathrm{mg} / \mathrm{kg}$ of $\mathrm{Zn}^{2+}$ ions in each injection. 
Table S1. Comparison of relaxivities of $\mathrm{T}_{1}$ contrast agents based on $\mathrm{Gd}^{3+}$-ligand complexes.

\begin{tabular}{|c|c|c|c|c|}
\hline Composition & $\mathbf{r}_{1}\left(\mathrm{mM}^{-1} \mathbf{s}^{-1}\right)$ & $\mathbf{r}_{2}\left(\mathrm{mM}^{-1} \mathrm{~s}^{-1}\right)$ & $\mathbf{r}_{2} / \mathbf{r}_{1}$ & Field(T) \\
\hline Gd-AuNC ${ }^{1}$ & 41.5 & 49.6 & 1.2 & 0.55 \\
\hline GO-DTPA-Gd ${ }^{2}$ & 10.8 & & & 11.7 \\
\hline h-P(CPTM-co-DOTA-(Gd))-b-P(OEGMA-co-GP & 3.12 & & & 1.5 \\
\hline \multicolumn{5}{|l|}{ MA) ${ }^{3}$} \\
\hline Added $2 \mu \mathrm{M}$ DTT in the above solution ${ }^{3}$ & 4.04 & & & 1.5 \\
\hline Added $10 \mathrm{mM}$ DTT in the above solution ${ }^{3}$ & 29.92 & & & 1.5 \\
\hline Added $20 \mathrm{mM}$ DTT in the above solution ${ }^{3}$ & 35.97 & & & 1.5 \\
\hline HSP-BP-DTPA-Gd ${ }^{4}$ & 25 & & & 0.73 \\
\hline gold nanorods-GdDTPAA ${ }^{5}$ & 21.3 & & & 9.4 \\
\hline PbdEO/PEOCL: Gd-DTPA-G3 ${ }^{6}$ & 7.5 & & & 1.41 \\
\hline Gd-TREN-bis-HOPO-TAM-ethylamine-EA ${ }^{7}$ & 38 & & & 1.41 \\
\hline Gd-TREN-bis-HOPO-TAM-ethylamine-PLL ${ }^{7}$ & 21 & & & 1.41 \\
\hline DNA-Gd ${ }^{\mathrm{III}} @$ AuNP-13 nm & 16.9 & & & 1.41 \\
\hline DNA-Gd ${ }^{\mathrm{III}} @$ AuNP-30 nm ${ }^{8}$ & 20 & & & 1.41 \\
\hline DNA-Gd ${ }^{\mathrm{III} 8}$ & 8.7 & & & 1.41 \\
\hline MS2-HOPO-Lin ${ }^{9}$ & 29.7 & & & 1.41 \\
\hline MS2-HOPO-SS ${ }^{9}$ & 38.2 & & & 1.41 \\
\hline MS2-HOPO-RR ${ }^{9}$ & 25.4 & & & 1.41 \\
\hline Gd-DTPA (this work) & 3.7 & 4.2 & 1.1 & 0.55 \\
\hline $\mathrm{GdCl}_{3}$ (this work) & 8.12 & 9.28 & 1.14 & 0.55 \\
\hline
\end{tabular}


Table S2. Comparison of relaxivities of $T_{1}$ contrast agents based on inorganic nanoparticles.

\begin{tabular}{|c|c|c|c|c|}
\hline Composition & $\mathbf{r}_{1}\left(\mathrm{mM}^{-1} \mathrm{~s}^{-1}\right)$ & $\mathbf{r}_{2}\left(\mathrm{mM}^{-1} \mathrm{~s}^{-1}\right)$ & $\mathbf{r}_{2} / \mathbf{r}_{1}$ & Field (T) \\
\hline GHC-1 $1^{10}$ & 34.8 & 40.6 & 1.17 & 0.55 \\
\hline $\mathrm{a}-\mathrm{NaGdF}_{4}: \mathrm{Yb}^{3+}: \mathrm{Er}^{3+} / \mathrm{NaGdF}_{4}{ }^{11}$ & 1.4 & & & 1.5 \\
\hline $\mathrm{PGP} /$ dextran-K01 ${ }^{12}$ & 13.9 & 15.0 & 1.1 & 0.47 \\
\hline $\mathrm{Gd}_{0.6} \mathrm{Eu}_{0.4} \mathrm{VO}_{4} / \mathrm{SiO}_{2}{ }^{13}$ & 2.52 & 3.03 & 1.2 & \\
\hline $\mathrm{Gd}_{0.6} \mathrm{Eu}_{0.4} \mathrm{VO}_{4}{ }^{13}$ & 2.97 & 3.47 & 1.17 & \\
\hline $\mathrm{GdVO}_{4}{ }^{13}$ & 0.95 & 1.31 & 1.08 & \\
\hline $\mathrm{GdVO}_{4}{ }^{\mathrm{cit13}}$ & 4.6 & 5.52 & 1.2 & \\
\hline $\mathrm{Gd}_{0.6} \mathrm{Eu}_{0.4} \mathrm{VO}_{4}{ }^{\mathrm{cit} 13}$ & 8.18 & 9.38 & 1.15 & \\
\hline GadoSiPEG $^{14}$ & 8.8 & 11.4 & 1.3 & 7 \\
\hline $\mathrm{Gd}_{2} \mathrm{O}_{3}{ }^{15}$ & 9.9 & 10.5 & 1.1 & 1.5 \\
\hline $\mathrm{HMnO} @ \mathrm{mSiO}_{2}{ }^{16}$ & 1.72 & 11.3 & 6.6 & 1.5 \\
\hline $\mathrm{HMONs}^{17}$ & 1.42 & 7.74 & 5.5 & 3.0 \\
\hline Core@ $\mathrm{NaGdF}_{4}{ }^{18}$ & 6.18 & & & 3.0 \\
\hline $\mathrm{KGdF}_{4}{ }^{19}$ & 5.86 & & & 9.4 \\
\hline $\mathrm{UCNPs}^{20}$ & 1.40 & & & 1.5 \\
\hline 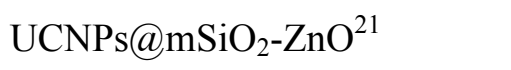 & 4.78 & & & 1.5 \\
\hline Gd-doped $\mathrm{ZnO} \mathrm{QDs}^{22}$ & 16 & & & 1.5 \\
\hline ZnO-Gd (this work) & 49.5 & 63 & 1.27 & 0.55 \\
\hline ZnO-Gd-DOX (this work) & 52.5 & 66.5 & 1.26 & 0.55 \\
\hline
\end{tabular}

\section{References}

1 Liang G. H.; Ye D. X.; Zhang X. X.; Dong F.; Chen H.; Zhang S.; Li J. Q.; Shen X. R.; Kong J. L. One-Pot Synthesis of $\mathrm{Gd}^{3+}$-Functionalized Gold Nanoclusters for Dual Model (Fluorescence/Magnetic Resonance) Imaging. J. Mater. Chem. B 2013, 1, 3545.

2 Zhang M. X.; Cao Y. H.; Chong Y.; Ma Y. F.; Zhang H. L.; Deng Z. W.; Hu C. H.; Zhang Z. J. Graphene Oxide Based Theranostic Platform for $\mathrm{T}_{1}$-Weighted Magnetic Resonance Imaging and Drug Delivery. ACS Appl. Mater. Inter. 2013, 5, 13325. 
3 Liepold L. O.; Abedin M. J.; Buckhouse E. D.; Frank J. A.; Young M. J.; Douglas T. Supramolecular Protein Cage Composite MR Contrast Agents with Extremely Efficient Relaxivity Properties. Nano Lett. 2009, 9, 4520 .

4 Kim H. K.; Park J. A.; Kim K. M.; Md N. S.; Kang D. S.; Lee J.; Chang Y.; Kim T. J. Gd-Complexes of Macrocyclic DTPA Conjugates of 1,10 -Bis(amino)Ferrocenes as High Relaxivity MRI Blood-Pool Contrast Agents (BPCAs). Chem. Comm. 2010, 46, 8442.

5 Sun H. M.; Yuan Q. H.; Zhang B. H.; Ai K. L.; Zhang P. G.; Lu L. H.; Gd ${ }^{\text {III }}$ Functionalized Gold Nanorods for Multimodal Imaging Applications. Nanoscale 2011, 3, 1990.

6 Cheng Z. L.; Thorek D.; Tsourkas A. Porous Polymersomes with Encapsulated Gd-Labeled Dendrimers as Highly Efficient MRI Contrast Agents. Adv. Funct. Mater. 2009, 19, 3753.

7 Floyd W. C.; Klemm P. J.; Smiles D. E.; Kohlgruber A. C.; Pierre V. C.; Mynar J. L.; Frechet J.; Raymond K. N. Conjugation Effects of Various Linkers on Gd(III) MRI Contrast Agents with Dendrimers: Optimizing the Hydroxypyridinonate (HOPO) Ligands with Nontoxic, Degradable Esteramide (EA) Dendrimers for High Relaxivity. J. Am. Chem. Soc. 2011, 133, 2390.

8 Song Y.; Xu X. Y.; MacRenaris K. W.; Zhang X. Q.; Mirkin C. A.; Meade T. J. Multimodal Gadolinium-Enriched DNA-Gold Nanoparticle Conjugates for Cellular Imaging. Angew. Chem. Int. Ed. 2009, 48, 9143 .

9 Garimella P. D.; Datta A.; Romanini D. W.; Raymond K. N.; Francis M. B. Multivalent, High-Relaxivity MRI Contrast Agents Using Rigid Cysteine-Reactive Gadolinium Complexes. J. Am. Chem. Soc. 2011, 133, 14704.

10 Liang G. H.; Cao L. L.; Chen H.; Zhang Z. Y.; Zhang S.; Yu S. N.; Shen X. R.; Kong J. L. Ultrasmall Gadolinium Hydrated Carbonate Nanoparticle: An Advanced $\mathrm{T}_{1}$ MRI Contrast Agent with Large Longitudinal Relaxivity. J. Mater. Chem. B 2013, 1, 629.

11 Park Y. I.; Kim J. H.; Lee K. T.; Jeon K. S.; Na H. Bin; Yu J. H.; Kim H. M.; Lee N.; Choi S. H., Baik S. I.; Kim H.; Park S. P.; Park B. J.; Kim Y. W.; Lee S. H.; Yoon S. Y.; Song I. C.; Moon W. K.; Suh Y. D.; Hyeon T.; Nonblinking and Nonbleaching Upconverting Nanoparticles as an Optical Imaging Nanoprobe and $\mathrm{T}_{1}$ Magnetic Resonance Imaging Contrast Agent. Adv. Mater. 2009, $21,4467$.

12 Hifumi H.; Yamaoka S.; Tanimoto A.; Citterio D.; Suzuki K. Gadolinium-Based Hybrid Nanoparticles as a Positive MR Contrast Agent. J. Am. Chem. Soc. 2006, 128, 15090.

13 Abdesselem M.; Schoeffel M.; Maurin I.; Ramodiharilafy R.; Autret G.; Clement O.; Tharaux P. L.; Boilot J. P.; Gacoin T.; Bouzigues C.; Alexandrou A. Multifunctional Rare-Earth Vanadate Nanoparticles: Luminescent Labels, Oxidant Sensors, and MRI Contrast Agents. ACS Nano 2014, 8, 11126.

14 Bridot J. L.; Faure A. C.; Laurent S.; Riviere C.; Billotey C.; Hiba B.; Janier M.; Josserand V.; Coll J. L.; Elst L. Vander; Muller R.; Roux S.; Perriat P.; Tillement O. Hybrid Gadolinium Oxide Nanoparticles: Multimodal Contrast Agents for in Vivo Imaging. J. Am. Chem. Soc. 2007, 129, 5076.

15 Park J. Y.; Baek M. J.; Choi E. S.; Woo S.; Kim J. H.; Kim T. J.; Jung J. C.; Chae K. S.; Chang Y.; Lee G. H. Paramagnetic Ultrasmall Gadolinium Oxide Nanoparticles as Advanced $T_{1}$ MRI Contrast Agent: Account for Large Longitudinal Relaxivity, Optimal Particle Diameter, and In Vivo $\mathrm{T}_{1} \mathrm{MR}$ Images. ACS Nano 2009, 3, 3663.

16 Kim T.; Momin E.; Choi J.; Yuan K.; Zaidi H.; Kim J.; Park M.; Lee N.; McMahon M. T.; Quinones-Hinojosa A.; Bulte J.; Hyeon T.; Gilad A. A. Mesoporous Silica-Coated Hollow Manganese Oxide Nanoparticles as Positive $\mathrm{T}_{1}$ Contrast Agents for Labeling and MRI Tracking of Adipose Derived Mesenchymal Stem Cells. J. Am. Chem. Soc. 2011, 133, 2955. 
17 Shin J. M.; Anisur R. M.; Ko M. K.; Im G. H.; Lee J. H.; Lee I. S. Hollow Manganese Oxide Nanoparticles as Multifunctional Agents for Magnetic Resonance Imaging and Drug Delivery. Angew. Chem. Int. Ed. 2009, 48, 321.

18 Chen F.; Bu W. B.; Zhang S. J.; Liu X. H.; Liu J. N.; Xing H. Y.; Xiao Q. F.; Zhou L. P.; Peng W. J.; Wang L. Z.; Shi J. L. Positive and Negative Lattice Shielding Effects Co-existing in Gd (III) Ion Doped Bifunctional Upconversion Nanoprobes. Adv. Funct. Mater. 2011, 21, 4285.

19 Ju Q.; Tu D. T.; Liu Y. S.; Li R. F.; Zhu H. M.; Chen J. C.; Chen Z.; Huang M. D.; Chen X. Y. Amine-Functionalized Lanthanide-Doped $\mathrm{KGdF}_{4}$ Nanocrystals as Potential Optical/Magnetic Multimodal Bioprobes. J. Am. Chem. Soc. 2012, 134, 1323.

20 Park Y. I.; Kim J. H.; Lee K. T.; Jeon K. S.; Na H. Bin; Yu J. H.; Kim H. M.; Lee N.; Choi S. H.; Baik S. I.; Kim H.; Park S. P.; Park B. J.; Kim Y. W.; Lee S. H.; Yoon S. Y.; Song I. C.; Moon W. K.; Suh Y. D.; Hyeon T. Nonblinking and Nonbleaching Upconverting Nanoparticles as an Optical Imaging Nanoprobe and T1 Magnetic Resonance Imaging Contrast Agent. Adv. Mater. 2009, 21, 4467.

21 Wang Y. H.; Song S. Y.; Liu J. H.; Liu D. P.; Zhang H. J. ZnO-Functionalized Upconverting Nanotheranostic Agent: Multi-Modality Imaging-Guided Chemotherapy with On-Demand Drug Release Triggered by pH. Angew. Chem. Int. Ed. 2015, 54, 536.

22 Liu Y. L.; Ai K. L.; Yuan Q. H.; Lu L. H. Fluorescence-Enhanced Gadolinium-Doped Zinc Oxide Quantum Dots for Magnetic Resonance and Fluorescence Imaging. Biomaterials 2011, 32, 1185. 